\title{
Paeoniflorin inhibits proliferation and induces apoptosis of human glioma cells via microRNA-16 upregulation and matrix metalloproteinase-9 downregulation
}

\author{
WEIHUA LI ${ }^{1}$, ZHONGHUA QI ${ }^{1}$, ZHENQING WEI ${ }^{1}$, SIWEI LIU ${ }^{2}$, \\ PIN WANG ${ }^{2}$, YANWEI $\mathrm{CHEN}^{3}$ and YONGSHUN ZHAO ${ }^{1}$ \\ ${ }^{1}$ Department of Neurosurgery, 1st Affiliated Hospital of Dalian Medical University, Dalian, Liaoning 116011; \\ ${ }^{2}$ Academic Affairs Office, Dalian Medical University, Dalian, Liaoning 116044; ${ }^{3}$ Department of Pharmacy, \\ 1st Affiliated Hospital of Dalian Medical University, Dalian, Liaoning 116011, P.R. China
}

Received August 25, 2014; Accepted March 23, 2015

DOI: $10.3892 / \mathrm{mmr} .2015 .3718$

\begin{abstract}
Paeoniflorin is one of the active ingredients of the commonly used herbal medicine derived from Paeonia, which exhibits anticancer properties. MicroRNA-16 (miR-16) is upregulated in $\mathrm{CD}^{-3} 3^{-}$cells, but downregulated in $\mathrm{CD}^{-133^{+}}$ cells from glioma tissue. Matrix metalloproteinase-9 (MMP-9) expression in glioma tissue samples is significantly higher than that in healthy brain tissue samples. Therefore, miR-16 and MMP-9 expression may be associated with glioma pathogenesis. In the present study, the effects of paeoniflorin on glioma were analyzed. U87 cells were treated with paeoniflorin at 0 , 5, 10 and $20 \mu \mathrm{M}$ concentrations. The results suggested that paeoniflorin inhibited U87 cell proliferation and accelerated cell apoptosis. In the present study paeoniflorin treatment increased miR-16 expression and reduced MMP-9 protein expression in U87 cells. Additionally, the results of the present study suggested that miR-16 may regulate MMP-9 expression in miR-16-transfected U87 cells. Furthermore, anti-miR-16 antibodies were used in order to investigate the apoptotic effects of paeoniflorin on U87 cells. The results demonstrated that paeoniflorin inhibits proliferation and induces apoptosis of human glial cells, via miR-16 upregulation and MMP-9 downregulation.
\end{abstract}

\section{Introduction}

Glioma is the most common type of intracranial neuroepithelial tumor and the most aggressive primary tumor, exhibiting rapid growth rates (1). Furthermore, the 2-year survival rate for

Correspondence to: Dr Yongshun Zhao, Department of Neurosurgery, 1st Affiliated Hospital of Dalian Medical University, 222 Zhong Shan Road, Dalian, Liaoning 116011, P.R. China

E-mail: yongshunzhaomr@163.com

Key words: paeoniflorin, glioma, matrix metalloproteinases-9, microRNA-16 patients with poorly differentiated glioma is only $10 \%(2,3)$. Glioma accounts for $44.6 \%$ of tumors in the central nervous system, with high recurrence and mortality rates (4). Survival times are low and mortality rates are high in patients with glioma, and the disease is associated with poor prognosis (5). Furthermore, the outcomes of radiotherapy treatment combined with chemotherapy do not improve patient prognosis (6).

Expression profiling analysis is an effective method used to demonstrate abnormalities in miRNA expression patterns (7). Glioma exhibits a unique miRNA expression profile, which distinguishes it from the surrounding healthy brain tissue. Furthermore, miRNA expression profiles vary between the different stages of glioma (8). Compared with healthy brain tissue, 17 miRNAs, including miR-21, -221, -222, $-125 \mathrm{~b}$ and $-10 \mathrm{~b}$, are overexpressed in glial cells, whereas 33 miRNAs, including miR-181a/b/c, -124, -137, -7 and -128, are downregulated in glial cells (9). Furthermore, six miRNAs (miR-16, -107, -185, -425, -451 and -486) are upregulated in CD133- cells, but downregulated in tumorous glial stem cells $\left(\mathrm{CD} 133^{+}\right)$. Research has demonstrated that miR-16 expression is markedly decreased in glioma cell lines compared with healthy cells and that the upregulation of miR-16 may suppress glioma growth and invasiveness $(10,11)$.

Transcription and expression levels of matrix metalloproteinase (MMP)-2 and -9 are associated with the degree of malignancy in glioma (12). MMP-2 and -9 may be used as an indicator of malignant human brain glioma (13). MMP expression predominantly modulates the local invasiveness of glial cells. Therefore, MMP-2 and -9 expression may reflect the degradation of glima matrix (14). Studies have demonstrated that U251 multiform glioblastoma expresses MMP-9 during cancer cell invasion $(15,16)$. In addition, MMP tissue inhibitor treatment is capable of decreasing percentage cell invasion from 42 to $10 \%$ (17).

Paeoniflorin is an active ingredient of the commonly used herbal medicine derived from Paeonia (18). Pharmacological studies have demonstrated that paeoniflorin prevents free radical damage, inhibits intracellular calcium-overload and exhibits anticancer activities, as well as exhibiting a number of biological effects, such as inhibiting cancer cell 
proliferation, improving microcirculation, an prevents oxidization and convulsion (19). Paeoniflorin treatment induces human cervical cancer cell apoptosis via the upregulation of the pro-apoptotic genes, Bax and caspase-3, and the downregulation of the anti-apoptotic gene, Bcl-2 (20). Paeoniflorin inhibits $\mathrm{H}_{2} \mathrm{O}_{2}$-induced apoptosis in SH-SY5Y cells, reducing $\mathrm{H}_{2} \mathrm{O}_{2}$-induced MMP expression changes. Paeoniflorin treatment inhibits cluster of differentiation 147 expression in THP-1 cells and reduces MMP-9 secretion. In a previous study, the levels of transforming growth factor $\beta 1$ and thymidylate synthetase were significantly higher in healthy samples compared with paeoniflorin-treated samples, which corresponded with an improvement in sample histology. By contrast, MMP-2 and -9 expression levels demonstrated the opposite results (21).

It is hypothesized that paeoniflorin may be useful for the treatment of glioma. The present study investigated the molecular mechanisms underlying the effects of paeoniflorin on glial cells. In order to test this hypothesis, the effects of different concentrations of paeoniflorin treatment on human glioma cells were analyzed.

\section{Materials and methods}

Primary reagents. The chemical structure of paeoniflorin is indicated in Fig. 1. Paeoniflorin (98\%; Sigma-Aldrich, St. Louis, MO, USA) was dissolved in physiological saline solution. Dulbecco's modified Eagle's medium (DMEM), fetal calf serum and Lipofectamine $2000{ }^{\circledR}$ were purchased from Invitrogen Life Technologies (Carlsbad, CA, USA). 3-[4,5-dimethylthiazol-2-thiazolyl]-2,5-diphenyl-tetrazolium bromide (MTT) was purchased from Beyotime Institute of Biotechnology (Haimen, China).

Cancer cell lines. The U87 glioma cell line was purchased from the cell bank of the Chinese academy of sciences (Shanghai, China). U87 cells were cultured in DMEM, supplemented with $10 \%$ fetal calf serum, $100 \mathrm{U} / \mathrm{ml}$ penicillin and $100 \mathrm{mg} / \mathrm{ml}$ streptomycin at $37^{\circ} \mathrm{C}$ and $5 \% \mathrm{CO}_{2}$.

MTT viability assay. U87 cells $\left(5.0 \times 10^{3}\right.$ cells/well) were seeded in 96-well culture clusters and incubated at $37^{\circ} \mathrm{C}$ and $5 \% \mathrm{CO}_{2}$ in a humidified incubator, for $24 \mathrm{~h}$. Following treatment with different concentrations of paeoniflorin $(0,5,10$ and $20 \mu \mathrm{M})$, cell viability was measured using an MTT assay. MTT $(\sim 10-\mu 1 ; 10 \mathrm{mg} / \mathrm{ml})$ was added into each well and the wells were incubated at $37^{\circ} \mathrm{C}$ and $5 \% \mathrm{CO}_{2}$, for $4 \mathrm{~h}$. Subsequently, $200 \mu \mathrm{l}$ dimethylsulfoxide was added to each well. The wells were then agitated for $10 \mathrm{~min}$ at room temperature. Viable cells were detected using an enzyme-linked immunosorbent assay reader (SpectraMax ${ }^{\circledR}$ M5e, BioTek, USA) at $570 \mathrm{~nm}$.

Caspase-3 activity measurement. U87 cells (5.0x10 3 cells/well) were seeded in 96 -well culture clusters and incubated at $37^{\circ} \mathrm{C}$ and $5 \% \mathrm{CO}_{2}$ in a humidified incubator for $24 \mathrm{~h}$. Following treatment with paeoniflorin, A549 cells (Cell Bank of the Chinese Academy of Sciences, Shanghai, China) were centrifuged at $16,000 \mathrm{x} \mathrm{g}$ for $15 \mathrm{~min}$ at $4^{\circ} \mathrm{C}$. Caspase- 3 activity of cells was measured using a colorimetric caspase- 3 assay kit (Beyotime Institute of Biotechnology). Protein extracts

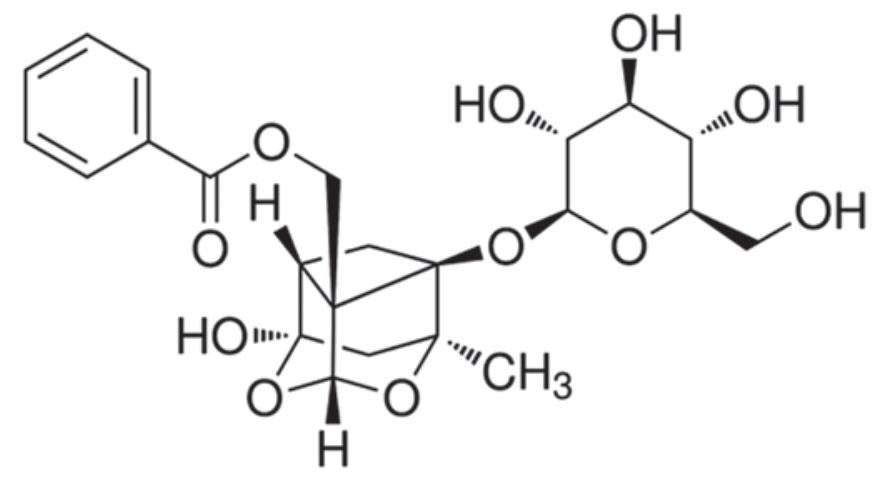

Figure 1. Chemical structure of paeoniflorin.

$(50-\mu \mathrm{g})$ were obtained from U87 cells and were incubated and added to a reaction buffer (Tianjin Hualida Biotechnology Co., Ltd., Tianjin, China), containing $85 \mu \mathrm{l}$ assay buffer and $10 \mu \mathrm{l}$ caspase-3 substrate (Ac-DEVD-pNA) at $37^{\circ} \mathrm{C}$ for $4-6 \mathrm{~h}$. The change was calculated at $405 \mathrm{~nm}$ using a microplate spectrophotometer (BioTek Instruments, Inc., Winooski, VT, USA).

Apoptosis assay. Flow cytometry (BD Biosciences, Franklin Lakes, NJ, USA) was conducted in order to investigate whether paeoniflorin treatment induced U87 cell apoptosis. Following treatment with paeoniflorin, A549 cells were collected and washed twice with phosphate-buffered saline (PBS). Annexin V-fluorescein isothiocyanate (FITC; $5 \mu \mathrm{l}$; BD Pharmingen, San Diago, CA, USA) was added to the A549 cells and stained using a binding buffer for $30 \mathrm{~min}$ in the dark according to the manufacturer's instructions. Subsequently, $10 \mu \mathrm{l}$ propidium iodide (PI) was added to the cells and incubated for $15 \mathrm{~min}$ at room temperature in the dark. Samples were then analyzed using flow cytometry (FACS Calibur; BD Biosciences).

MMP-9 expression. Gelatin zymography assays were used in order to investigate whether paeoniflorin inhibits MMP-9 expression in U87 cells. Following treatment with paeoniflorin, U87 cells were harvested and MMP-9 protein was electrophoresed on a $10 \%$ SDS-PAGE, containing $1 \%$ gelatin. Following gel electrophoresis, the gel was washed in $1.5 \%$ Triton X-100 (Shanghai Biological Co., Ltd., Shanghai, China) for $0.5-1 \mathrm{~h}$ and then washed in water. Gels were incubated in buffer ( $\mathrm{pH} \mathrm{8.0)}$ at $37^{\circ} \mathrm{C}$ for $12 \mathrm{~h}$. Gels were then stained with $0.2 \%$ Coomassie Brilliant Blue R-250 dye (Qingdao Jacob Chemical Reagent Sales Co., Ltd., Shandong, China) for $1 \mathrm{~h}$. MMP-9 protein expression was then quantified using a MiniBis system (DNR Bio-Imaging Systems Ltd., Jerusalem, Israel) and prestained SDS-PAGE standards (Hou-Bio Tech. Ltd., Shandong, China).

Reverse transcription-quantitative polymerase chain reaction (RT-qPCR) of miR-16 expression. RT-qPCR was used in order to investigate whether paeoniflorin treatment induced miR-16 expression in U87 cells. Following treatment with paeoniflorin, total RNA was extracted from the cells using TRIzol ${ }^{\circledR}$ reagent according to manufacturer's instructions (Invitrogen Life Technologies). SuperScript ${ }^{\circledR}$ III Reverse Transcriptase (Invitrogen Life Technologies) was used to 

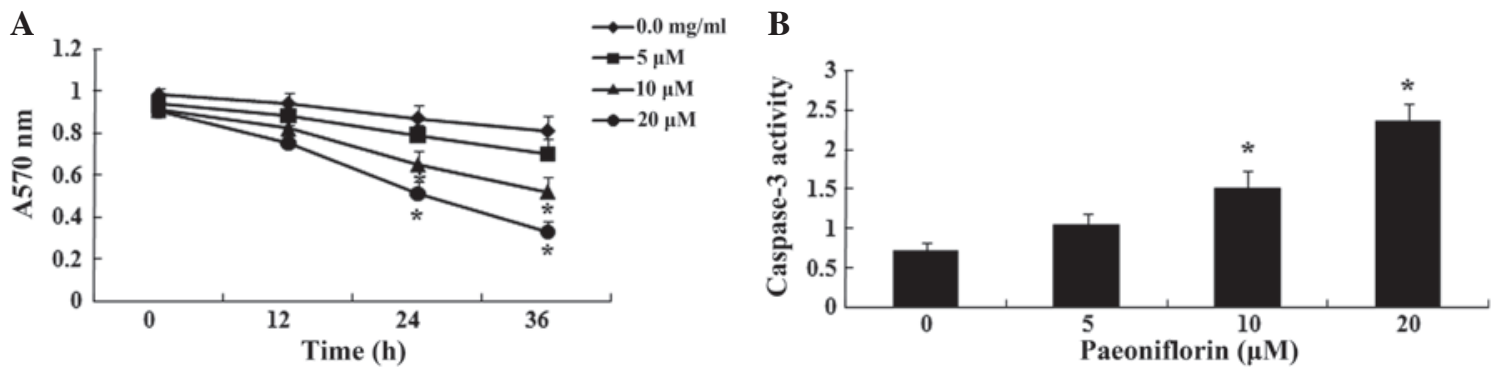

Figure 2. 3-(4,5-Dimethylthiazol-2-thiazolyl)-2,5-diphenyl-tetrazolium bromide analysis and caspase-3 activity. (A) Paeoniflorin treatment inhibited U87 cell growth in a dose-dependent manner and (B) paeoniflorin treatment led to a significant increase in caspase- 3 activity. "P $<0.05$ compared with the $0 \mu \mathrm{M}$ paeoniflorin treatment group.
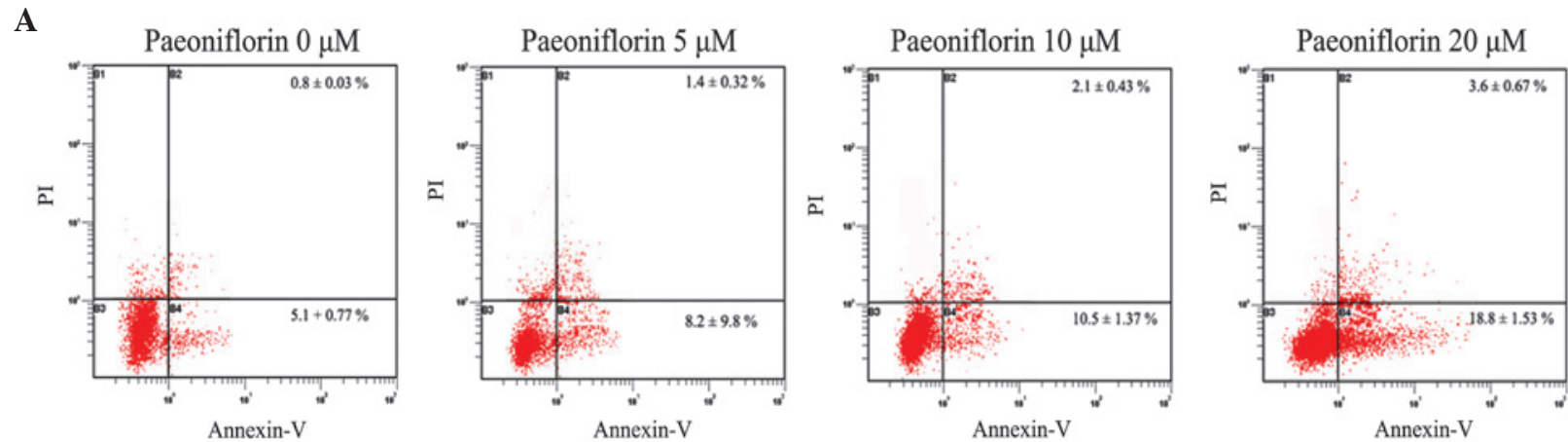

B

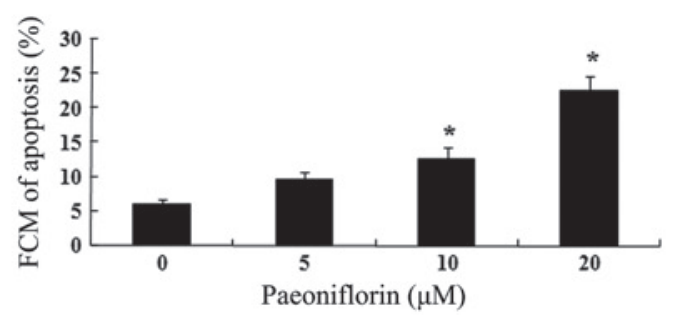

Figure 3. Flow cytometric analysis of cell apoptosis. (A) U87 cell apoptosis increased following paeoniflorin treatment for $24 \mathrm{~h}$ in a dose-dependent manner, according to Annexin V-fluorescein isothiocyanate/PI and (B) U87 cell apoptosis following paeoniflorin treatment. * $\mathrm{P}<0.05$ compared with the $0 \mu \mathrm{M}$ paeoniflorin treatment group. PI, propidium iodide.

analyze cDNA. and subsequently, $\mathrm{SYBR}^{\circledR}$ Green PCR Master mix (Life Technologies, Grand Island, NY, USA) was used to obtain the final cDNA. miR-16 mRNA expression was quantified using an RT-PCR kit (Invitrogen Life Technologies) according to the manufacturer's instructions and an 7900HT Real-time PCR detection system. The following primers were used: 5'-TAGCAGCACGTAAATATTGGC-3' for miR-16; 5'-TGGTGTCGTGGAGTCG-3' for $\beta$-actin; U6, forward 5'-CGCTTCGGCACATATACTA-3' and reverse 5'-CGCTTCACGAATTTGCGTGTCA-3'. The cycling conditions were as follows: $94^{\circ} \mathrm{C}$ for $10 \mathrm{~min}, 35$ cycles of $94^{\circ} \mathrm{C}$ for $30 \mathrm{sec}, 60^{\circ} \mathrm{C}$ for $30 \mathrm{sec}$ and $72^{\circ} \mathrm{C}$ for $30 \mathrm{sec}$, followed by $73^{\circ} \mathrm{C}$ for $5 \mathrm{~min}$.

miR-16 expression and anti-miR-16 transfection. miR-16 precursor and anti-miR-16 (Ambion Life Technologies, Carlsbad, CA, USA) were obtained from Sangon Biotech Co., Ltd. (Shanghai, China). U87 cells $\left(5 \times 10^{5}\right.$ cells/well $)$ were cultured in 6 well plates and transfected with miR-16 precursor/anti-miR-16 (Ambion Life Technologies) using
Lipofectamine 2000 (Invitrogen Life Technologies, Carlsbad, CA, USA) for $6 \mathrm{~h}$, following treatment with $10 \mu \mathrm{M}$ peaoniflorin for $24 \mathrm{~h}$. Subsequently, the transfection medium was replaced with DMEM containing 10\% fetal bovine serum without antibiotic (Beijing Genetic Company, Beijing, China) in a humidified atmosphere at $37^{\circ} \mathrm{C}$ with $5 \% \mathrm{CO}_{2}$ for $18 \mathrm{~h}$.

Statistical analysis. Experiments were performed at least three times and data are provided as the mean \pm standard error. Data were analyzed by Student's t-test using SPSS 17.0 software (SPSS, Inc,. Chicago, IL, USA). P $<0.05$ was considered to indicate a statistically significant difference.

\section{Results}

MTT analysis and caspase-3 activity. In order to determine the effects of paeoniflorin on U87 cells, U87 cell viability was analyzed following treatment with paeoniflorin $(0,5,10$ and $20 \mu \mathrm{M}$ ), using MTT assays. As shown in Fig. 2A, treatment with 10 and $20 \mu \mathrm{M}$ paeoniflorin for 24 or $36 \mathrm{~h}$ significantly reduced 


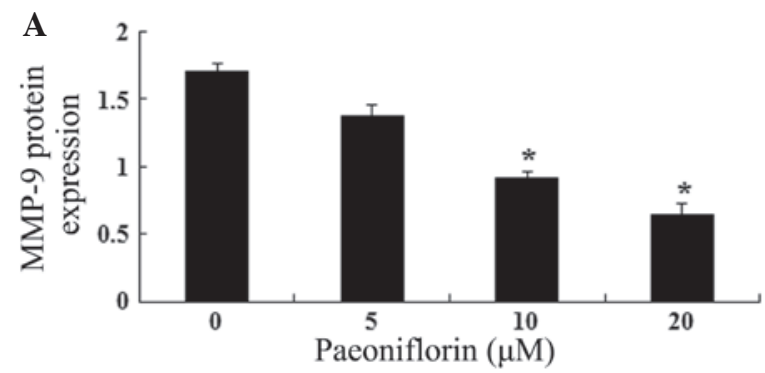

B

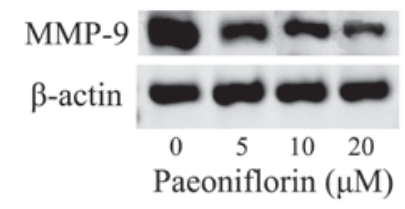

Figure 4. Paeoniflorin-induced inhibition of MMP-9 expression. (A) According to gelatin zymography assays, MMP-9 activity was reduced in U87 cells following treatment with paeoniflorin for $24 \mathrm{~h}$ in a dose-dependent manner. (B) MMP-9 protein expression. ${ }^{*} \mathrm{P}<0.05$ compared with the $0 \mu \mathrm{M}$ paeoniflorin treatment group. MMP-9, matrix metalloproteinase-9.

U87 cell viability compared with control cells $(\mathrm{P}<0.05)$. Cell viability decreased in a time- and concentration-dependent manner. Following paeoniflorin treatment $(0,5,10$ and $20 \mu \mathrm{M}$ ), caspase-3 activity in U87 cells was analyzed using a caspase-3 assay kit. As shown in Fig. 2B, treatment with 10 and $20 \mu \mathrm{M}$ paeoniflorin for $24 \mathrm{~h}$ significantly increased caspase- 3 activity in $\mathrm{U} 87$ cells, compared with $0 \mu \mathrm{M}$ treatment $(\mathrm{P}<0.05)$. Caspase- 3 activity increased in a concentration-dependent manner in U87 cells.

Flow cytometric analysis and cell apoptosis. In order to investigate the effect of paeoniflorin on cell apoptosis, U87 cells were treated with different concentrations of paeoniflorin for $24 \mathrm{~h}$. Flow cytometry assays demonstrated that paeoniflorin exerted a dose dependent inhibitory effect on U87 cell growth (Fig. 3A). As demonstrated in Fig. 3B, treatment with 10 and $20 \mu \mathrm{M}$ paeoniflorin for $24 \mathrm{~h}$ significantly increased the U87 cell apoptosis compared with the $0 \mu \mathrm{M}$ paeoniflorin-treated group $(\mathrm{P}<0.05)$.

Paeoniflorin-induced inhibition of MMP-9. In order to investigate the association between paeoniflorin-induced U87 cell growth inhibition and MMP-9 protein expression induction, gelatin zymography assays were conducted. As shown in Fig. 4A, the results of gelatin zymography assays suggested that paeoniflorin inhibited MMP-9 protein expression in a dose-dependent manner. As shown in Fig. 4B, treatment with 10 and $20 \mu \mathrm{M}$ paeoniflorin for $24 \mathrm{~h}$ significantly reduced the MMP-9 protein expression in U87 cells compared with control cells $(\mathrm{P}<0.05)$.

Paeoniflorin induces miR-16 expression. As shown in Fig. 5, paeoniflorin treatment promoted miR-16 expression levels in a dose-dependent manner. Treatment with 10 and $20 \mu \mathrm{M}$ paeoniflorin for $24 \mathrm{~h}$ significantly increased miR-16 expression levels in U87 cells, compared with control cells $(\mathrm{P}<0.05)$.

Overexpression of miR-16 and MMP-9 expression levels. In order to investigate the association between miR-16 expression and paeoniflorin-induced MMP-9 protein expression, an miR-16 precursor was transfected into U87 cells. As shown in Fig. 6A and B, miR-16 upregulation led to significant inhibition of MMP-9 protein expression.

Anti-miR-16 reverses the antitumor effects of paeoniflorin. An anti-miR-16 antibody was transfected into the U87 cells. The

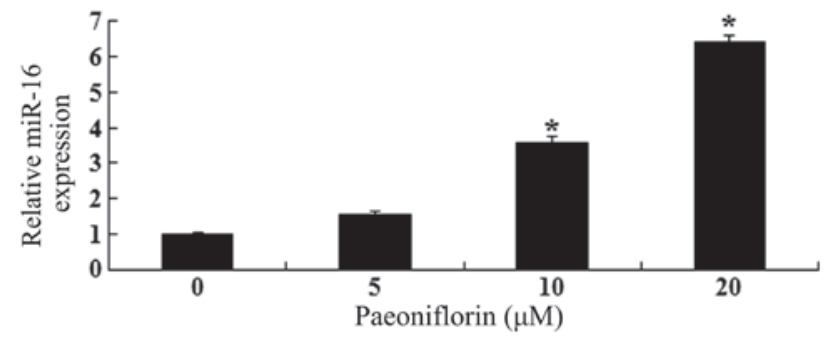

Figure 5. Paeoniflorin activated miR-16 expression. Paeoniflorin treatment increased the expression of miR-16 in a dose-dependent manner. ${ }^{*} \mathrm{P}<0.05$ compared with the $0 \mu \mathrm{M}$ paeoniflorin treatment group. miR, microRNA.

\section{A}

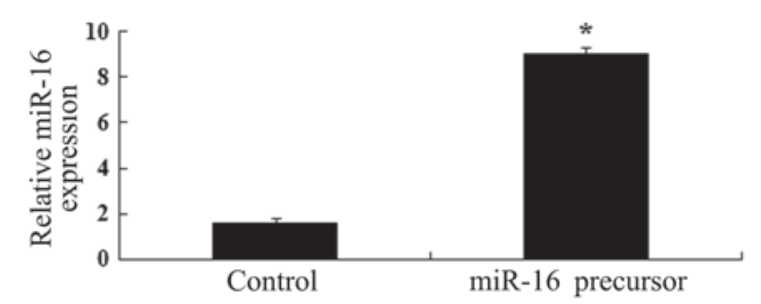

B

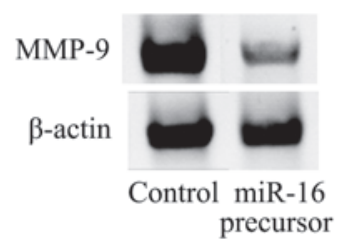

Figure 6. miR-16 overexpression and MMP-9 expression. (A) miR-16 precursor significantly elevated the expression of miR-16 and (B) transfection of miR-16 precursor led to a decrease in MMP-9 protein expression levels. ${ }^{*} \mathrm{P}<0.05$ compared with the $0 \mu \mathrm{M}$ paeoniflorin treatment group. miR, microRNA.

results indicated that miR-16 expression was significantly lower in anti-miR-16-transfected U87 cells compared with control cells (Fig.7A). The anti-miR-16 antibody significantly reduced the anticancer effects of paeoniflorin treatment $(10 \mu \mathrm{M})$ on U87 cell proliferation (Fig. 7B) and U87 cell apoptosis (Fig. 7C) at $24 \mathrm{~h}$. The results of the present study suggested that anti-miR-29b may influence the anticancer effects of paeoniflorin $(10 \mu \mathrm{M})$ via the downregulation of MMP-9 expression (Fig. 7D). 
A

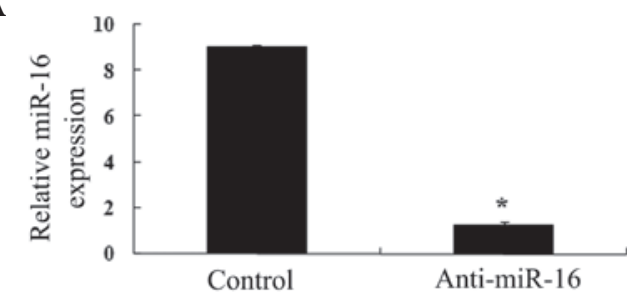

C

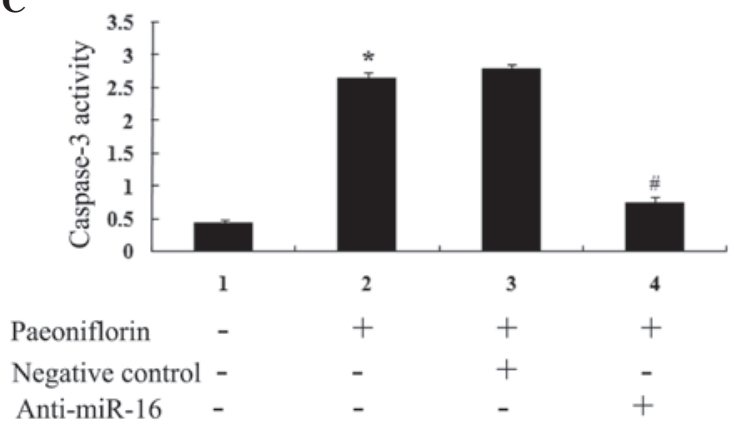

B

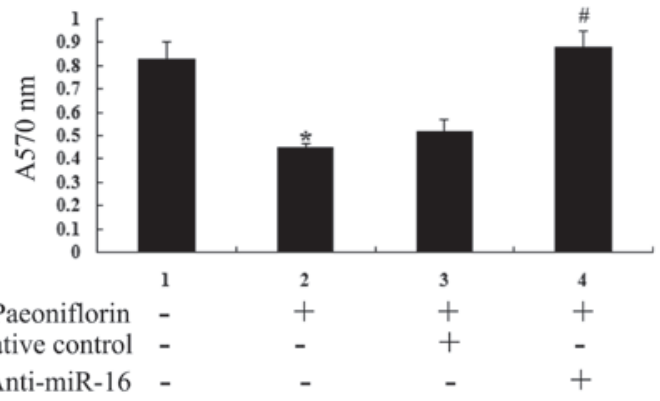

D

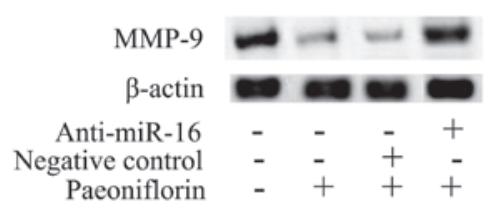

Figure 7. Anti-miR-16 reverses the antitumor effects of paeoniflorin. (A) Anti-miR-16 could significantly diminish the expression of miR-16 in U87 cells; (B) following treatment with paeoniflorin $(10 \mu \mathrm{M})$ for $24 \mathrm{~h}$, anti-miR-29b promoted cell proliferation; (C) following treatment with $10 \mu \mathrm{M}$ paeoniflorin for $24 \mathrm{~h}$, anti-miR-29b inhibited U87 cell apoptosis; and (D) anti-miR-16 significantly increased MMP-9 activity in U87 cells following $10 \mu \mathrm{M}$ paeoniflorin treatment at $24 \mathrm{~h}$. ${ }^{*} \mathrm{P}<0.05$ compared with the $0 \mu \mathrm{M}$ paeoniflorin treatment group, and ${ }^{\#} \mathrm{P}<0.01$ compared with the paeoniflorin-treated group transfected with negative control. miR, microRNA.

\section{Discussion}

Malignant glioma is the most predominant type of primary brain tumor in adults with relatively high rates of recurrences (22). Diffuse glioma cells are able to infiltrate the surrounding brain tissue, which is one of the most important characteristics of glioma (23). Therefore, novel approaches for glioma therapy are required.

Paeoniflorin, an active compound derived from the medicinal herb Paeonia, has been shown to exhibit a variety of biological effects (24). Paeoniflorin treatment may increase superoxide dismutase (SOD) levels and reduce malondialdehyde (MDA) content in ischemic brain tissue. It has been suggested that paeoniflorin treatment, following cerebral ischemia, may inhibit the production of free radicals, improve SOD activity and decrease MDA content in the brain. Therefore, paeoniflorin treatment may protect the brain from secondary neuron injury in patients with cerebral ischemia (25). A study has reported that paeoniflorin may modulate multidrug resistance of the human gastric cancer cell line, via the inhibition of nuclear factor (NF) $-\kappa B$ activation (26). Paeoniflorin treatment may decrease MMP-9 expression levels in human liver carcinoma cells. It inhibits human liver carcinoma cell growth, metastasis and invasion (27). The results of the present study suggested that paeoniflorin may be an effective agent for the inhibition of proliferation and induction of apoptosis in U87 cells.

The upregulation of MMP-9 expression and the downregulation of p16 expression in glioma may be associated with tumor invasiveness. MMP-9 expression was shown to be lower in non-malignant astrocytoma cells, than in anaplastic astrocytoma and glioblastoma multiforme cells exhibiting high levels of malignancy (28). High levels of MMP-9 expression may reflect the degree of malignancy and invasiveness in brain glioma. High MMP-9 expression and low phosphatase and tensin homolog expression levels are indicators of increased glioma invasiveness. The combination of the two indices may be used as an important reference for diagnosis and prognosis for patients with glioma $(14,29)$. The results of the present study demonstrated that paeoniflorin is associated with the expression of MMP-9 in U87 cells.

miRs are involved in the development of a number of diseases, including cancer. They are typically underexpressed in cancer tissues and the inhibition of the expression of certain miRs may lead to the occurrence of cancer. A small number of miRs are overexpressed in cancer tissues and are associated with tumor genes. However, the majority of miRs are underexpressed in tumor tissues, serving as tumor suppressor genes in cancer (30). A number of experiments have demonstrated the involvement of miR-16 as a tumor suppressor gene in glioma growth, via the inhibition of $\mathrm{Bcl} 2$ and the NF- $\kappa$ B1/MMP-9 signaling pathway (10).

In the present study, treatment of U87 cells with paeoniflorin resulted in a significant increase in miR-16 expression levels. The results of the present study suggested that upregulation of miR-16 promotes MMP-9 expression in U87 cells. Paeoniflorin treatment exerted anticancer effects against human glioma cells via upregulating miR-16 and downregulating MMP-9 expression. 
In conclusion, paeoniflorin may be useful for the treatment of human glioma. The results of the present study demonstrated that paeoniflorin treatment may lead to decreased proliferation and increased apoptosis of human glioma cells (31). To the best of our knowledge, the results of the present study support the hypothesis that paeoniflorin may be an effective antitumor agent for the treatment of human glioma (32). Paeoniflorin inhibited MMP-9 protein expression and promoted miR-16 expression in U87 cells. Upregulating miR-16 inhibited MMP-9 protein expression levels in anti-miR-16-transfected U87 cells. Therefore, miR-16 is associated with the downregulation of MMP-9 expression in U87 cells. Paeoniflorin treatment appeared to inhibit proliferation and accelerate apoptosis of human glioma cells via miR-16 upregulation and MMP-9 expression downregulation. To the best of our knowledge this is the first study to suggest that paeoniflorin may inhibit proliferation and accelerate apoptosis of human glioma cells via miR-16 upregulation and MMP-9 downregulation.

\section{Acknowledgements}

This study was supported by the National Natural Science Foundation of China (grant no. 81202964).

\section{References}

1. Huang Q, Zhang QB, Dong J, et al: Glioma stem cells are more aggressive in recurrent tumors with malignant progression than in the primary tumor, and both can be maintained long-term in vitro. BMC Cancer 8: 304, 2008.

2. Kim JK, Jin X, Sohn YW, Jin X, Jeon HY, Kim EJ, Ham SW, Jeon HM, Chang SY, Oh SY, et al: Tumoral RANKL activates astrocytes that promote glioma cell invasion through cytokine signaling. Cancer Lett 353: 194-200, 2014.

3. Liu B, Gao YQ, Wang XM, Wang YC and Fu LQ: Germacrone inhibits the proliferation of glioma cells by promoting apoptosis and inducing cell cycle arrest. Mol Med Rep 10: 1046-1050, 2014.

4. Janinis J, Efstathiou E, Panopoulos C, et al: Phase II study of temozolomide in patients with relapsing high grade glioma and poor performance status. Med Oncol 17: 106-110, 2000.

5. Nakano I: Therapeutic potential of targeting glucose metabolism in glioma stem cells. Expert Opin Ther Targets 18: 1233-1236, 2014.

6. Huber RM, Flentje M, Schmidt M, Pöllinger B, Gosse H, Willner J, Ulm K; Bronchial Carcinoma Therapy Group. Simultaneous chemoradiotherapy compared with radiotherapy alone after induction chemotherapy in inoperable stage IIIA or IIIB non-small-cell lung cancer: study CTRT99/97 by the Bronchial Carcinoma Therapy Group. J Clin Oncol. 2006 Sep 20;24(27):4397-404.

7. Godlewski J, Nowicki MO, Bronisz A, Williams S, Otsuki A, Nuovo G, Raychaudhury A, Newton HB, Chiocca EA and Lawler S: Targeting of the Bmi-1 oncogene/stem cell renewal factor by microRNA-128 inhibits glioma proliferation and self-renewal. Cancer Res 68: 9125-9130, 2008.

8. Silber J, Lim DA, Petritsch C, Persson AI, Maunakea AK, Yu M, Vandenberg SR, Ginzinger DG, James CD, Costello JF, et al: miR-124 and miR-137 inhibit proliferation of glioblastoma multiforme cells and induce differentiation of brain tumor stem cells. BMC Med 6: 14, 2008.

9. Calin GA and Croce CM: MicroRNA signatures in human cancers. Nat Rev Cancer 6: 857-866, 2006.

10. Yang TQ, Lu XJ, Wu TF, Ding DD, Zhao ZH, Chen GL, Xie XS, Li B, Wei YX, Guo LC, et al: MicroRNA-16 inhibits glioma cell growth and invasion through suppression of BCL2 and the nuclear factor- $\kappa$ B1/MMP9 signaling pathway. Cancer Sci 105: 265-271, 2014.
11. Li X, Ling N, Bai Y, Dong W, Hui GZ, Liu D, Zhao J and Hu J: MiR-16-1 plays a role in reducing migration and invasion of glioma cells. Anat Rec (Hoboken) 296: 427-432, 2013.

12. Gao H, Zhang S, Cao S, Yang Z, Pang Z and Jiang X: Angiopep-2 and activatable cell-penetrating peptide dual-functionalized nanoparticles for systemic glioma-targeting delivery. Mol Pharm 11: 2755-2763, 2014.

13. Nakano A, Tani E, Miyazaki K, Yamamoto Y and Furuyama J: Matrix metalloproteinases and tissue inhibitors of metalloproteinases in human gliomas. J Neurosurg 83: 298-307, 1995.

14. Rao JS, Steck PA, Tofilon P, Boyd D, Ali-Osman F, Stetler-Stevenson WG, Liotta LA and Sawaya R: Role of plasminogen activator and of $92-\mathrm{KDa}$ type IV collagenase in glioblastoma invasion using an in vitro matrigel model. J Neurooncol 18: 129-138, 1994.

15. Yan W, Zhang W, Sun L, et al: Identification of MMP-9 specific microRNA expression profile as potential targets of anti-invasion therapy in glioblastoma multiforme. Brain Res 1411: 108-115, 2011.

16. Wong ET, Alsop D, Lee D, et al: Cerebrospinal fluid matrix metalloproteinase-9 increases during treatment of recurrent malignant gliomas. Cerebrospinal Fluid Res 5: 1, 2008.

17. Ramaswamy P, Aditi Devi N, Hurmath Fathima K and Dalavaikodihalli Nanjaiah N: Activation of NMDA receptor of glutamate influences MMP-2 activity and proliferation of glioma cells. Neurol Sci 35: 823-829, 2014.

18. Zheng YQ, Wei W, Zhu L and Liu JX: Effects and mechanisms of Paeoniflorin, a bioactive glucoside from paeony root, on adjuvant arthritis in rats. Inflamm Res 56: 182-188, 2007.

19. Wang H, Zhou H, Wang CX, Li YS, Xie HY, Luo JD and Zhou Y: Paeoniflorin inhibits growth of human colorectal carcinoma HT 29 cells in vitro and in vivo. Food Chem Toxicol 50: 1560-1567, 2012.

20. Zhang L and Zhang S: Modulating Bcl-2 family proteins and caspase- 3 in induction of apoptosis by paeoniflorin in human cervical cancer cells. Phytother Res 25: 1551-1557, 2011.

21. Jiang Z, Chen W, Yan X, Bi L, Guo S and Zhan Z: Paeoniflorin protects cells from GalN/TNF- $\alpha$-induced apoptosis via ER stress and mitochondria-dependent pathways in human L02 hepatocytes. Acta Biochim Biophys Sin (Shanghai) 46: 357-367, 2014.

22. Gorlia T, Stupp R, Brandes AA, et al: New prognostic factors and calculators for outcome prediction in patients with recurrent glioblastoma: a pooled analysis of EORTC Brain Tumour Group phase I and II clinical trials. Eur J Cancer 48: 1176-1184, 2012.

23. Zhou GS, Song LJ and Yang B: Isoliquiritigenin inhibits proliferation and induces apoptosis of U87 human glioma cells in vitro. Mol Med Rep 7: 531-536, 2013.

24. No authors listed: Retraction: Pharmacokinetic interaction of paeoniflorin and sinomenine: Pharmacokinetic parameters and tissue distribution characteristics in rats and protein binding ability in vitro. J Pharmacol Sci 104: 283, 2007.

25. Yu J, Zhu X, Qi X, Che J and Cao B: Paeoniflorin protects human EA.hy 926 endothelial cells against gamma-radiation induced oxidative injury by activating the NF-E2-related factor $2 /$ heme oxygenase-1 pathway. Toxicol Lett 218: 224-234, 2013.

26. Fang S, Zhu W, Zhang Y, Shu Y and Liu P: Paeoniflorin modulates multidrug resistance of a human gastric cancer cell line via the inhibition of NF- $\kappa \mathrm{B}$ activation. Mol Med Rep 5: 351-356, 2012.

27. Lu JT, He W, Song SS and Wei W: Paeoniflorin inhibited the tumor invasion and metastasis in human hepatocellular carcinoma cells. Bratisl Lek Listy 115: 427-433, 2014.

28. Lee YD, Cui MN, Yoon HH, Kim HY, Oh IH and Lee JH: Down-modulation of Bis reduces the invasive ability of glioma cells induced by TPA, through $\mathrm{NF}-\kappa \mathrm{B}$ mediated activation of MMP-9. BMB Rep 47: 262-267, 2014.

29. Rao JS, Yamamoto M, Mohaman S, Gokaslan ZL, Fuller GN, Stetler-Stevenson WG, Rao VH, Liotta LA, Nicolson GL and Sawaya RE: Expression and localization of $92 \mathrm{kDa}$ type IV collagenase/gelatinase B (MMP-9) in human gliomas. Clin Exp Metastasis 14: 12-18, 1996.

30. Banno K, Iida M, Yanokura M, et al: MicroRNA in cervical cancer: OncomiRs and tumor suppressor miRs in diagnosis and treatment. ScientificWorldJournal 2014: 178075, 2014.

31. Zhang B, Pan X, Cobb GP and Anderson TA: microRNAs as oncogenes and tumor suppressors. Dev Biol 302: 1-12, 2007.

32. Wang F, Sun JY, Zhu YH, Liu NT, Wu YF and Yu F: MicroRNA-181 inhibits glioma cell proliferation by targeting cyclin B1. Mol Med Rep 10: 2160-2164, 2014. 